\title{
Embedding relations between local Hardy and modulation spaces
}

\author{
by \\ Masaharu Kobayashi (Tokyo), Akihiko Miyachi (Tokyo), \\ and Naонiтo Tomita (Osaka)
}

\begin{abstract}
A sharp embedding relation between local Hardy spaces and modulation spaces is given.
\end{abstract}

1. Introduction. The modulation spaces $M_{s}^{p, q}$ were introduced by Feichtinger [2-4] to measure smoothness of a function or distribution in a way different from Besov spaces, and they are now recognized as appropriate function spaces for time-frequency analysis (see Gröchenig $[8,9]$ ). To find simple necessary or sufficient conditions for a function or distribution to belong to a modulation space is one of the basic problems. For this purpose, embedding relations between modulation spaces and several other function spaces were studied. In particular, embedding relations between modulation spaces and Besov spaces were extensively studied (see Gröbner [7], Okoudjou [12], Toft [16], Sugimoto-Tomita [14] and Wang-Huang [19]). Although most of the early research on the modulation spaces $M_{s}^{p, q}$ was restricted to the case $1 \leq p, q \leq \infty$, they can also be extended to the case $0<p, q \leq \infty$ (see Triebel [17], Kobayashi [10] and Wang-Hudzik [20]). The purpose of this paper is to consider embedding relations between the modulation spaces $M_{s}^{p, q}$ with $p \leq 1$ and the local Hardy spaces $h^{p}$ of Goldberg [6].

Let $1 \leq p \leq \infty$, and let $p^{\prime}$ be the conjugate exponent of $p$ (that is, $\left.1 / p+1 / p^{\prime}=1\right)$. It is known that

$$
M^{p, \min \left\{p, p^{\prime}\right\}}\left(\mathbb{R}^{n}\right) \hookrightarrow L^{p}\left(\mathbb{R}^{n}\right) \hookrightarrow M^{p, \max \left\{p, p^{\prime}\right\}}\left(\mathbb{R}^{n}\right)
$$

(see, for example, [16, Proposition 1.7]). In this paper, we shall give a similar embedding relation with $L^{p}$ replaced by $h^{p}$, the local Hardy space. It should be pointed out that $h^{1} \hookrightarrow L^{1}$ and $h^{p}=L^{p}$ if $1<p<\infty$, and thus we shall treat the case $0<p \leq 1$. The following are our main results: 
Theorem 1.1. Let $0<p \leq 1,0<q \leq \infty$ and $s \in \mathbb{R}$. Then $M_{s}^{p, q}\left(\mathbb{R}^{n}\right) \hookrightarrow$ $h^{p}\left(\mathbb{R}^{n}\right)$ if and only if either of the following conditions is satisfied:

(a) $p \geq q$ and $s \geq 0$;

(b) $p<q$ and $s>n(1 / p-1 / q)$.

TheOREM 1.2. Let $0<p \leq 1,0<q \leq \infty$ and $s \in \mathbb{R}$. Then $h^{p}\left(\mathbb{R}^{n}\right) \hookrightarrow$ $M_{s}^{p, q}\left(\mathbb{R}^{n}\right)$ if and only if either of the following conditions is satisfied:

(c) $p>q$ and $s<-n(1 / p+1 / q-1)$;

(d) $p \leq q$ and $s \leq-n(1 / p+1 / q-1)$.

In the case $0<p \leq 1$ and $p \leq q \leq \infty$, Theorems 1.1 and 1.2 say that

$$
M^{p, p}\left(\mathbb{R}^{n}\right) \hookrightarrow h^{p}\left(\mathbb{R}^{n}\right) \hookrightarrow M_{-n(1 / p+1 / q-1)}^{p, q}\left(\mathbb{R}^{n}\right),
$$

and that these embeddings are in a sense optimal. Hence, for the embedding $h^{p} \hookrightarrow M_{s}^{p, \max \left\{p, p^{\prime}\right\}}$ to hold, the smoothness index $s$ must be negative in the case $0<p<1$, while we can take $s=0$ in the case $p \geq 1$. Here we understand that $p^{\prime}=\infty$ in the case $0<p<1$.

Theorem 1.2 also claims that $h^{1}\left(\mathbb{R}^{n}\right) \hookrightarrow M_{-n / q}^{1, q}\left(\mathbb{R}^{n}\right)$ if $1 \leq q \leq \infty$. This embedding should be compared with the fact that $L^{1}\left(\mathbb{R}^{n}\right) \hookrightarrow M_{-n / q}^{1, q}\left(\mathbb{R}^{n}\right)$ if $1 \leq q<\infty$ (see Remark 4.6).

It will be interesting to compare our result with the following result of Wang-Huang [19, Theorem 1.1]: if $0<p \leq 1$ and $0<q \leq \infty$, then

$$
M_{\max \{0, n(1 / p-1 / q)\}}^{p, q}\left(\mathbb{R}^{n}\right) \hookrightarrow B_{0}^{p, q}\left(\mathbb{R}^{n}\right) \hookrightarrow M_{-n(1 / p+1 / q-1)}^{p, q}\left(\mathbb{R}^{n}\right),
$$

where $B_{0}^{p, q}$ is the Besov space. Let $0<p \leq 1$. Firstly, since $B_{0}^{p, p}\left(\mathbb{R}^{n}\right) \hookrightarrow$ $h^{p}\left(\mathbb{R}^{n}\right)$, if $p=q$, then the right hand embedding of (1.1) improves the right hand embedding of (1.2). Secondly, if $q \geq 2$, then $h^{p}\left(\mathbb{R}^{n}\right) \hookrightarrow B_{0}^{p, q}\left(\mathbb{R}^{n}\right)$, and hence the right hand embedding of (1.2) covers the right hand embedding of (1.1). Thirdly, since $B_{0}^{p, p}\left(\mathbb{R}^{n}\right) \hookrightarrow h^{p}\left(\mathbb{R}^{n}\right)$, the left hand embedding of (1.1) follows from the left hand embedding of (1.2) with $p=q$. These observations follow from the fact that the local Hardy space $h^{p}$ coincides with the TriebelLizorkin space $F_{0}^{p, 2}$, and from the embedding relation between $F_{0}^{p, q}$ and $B_{0}^{p, q}$ (see [13] and [18, Chapter 2]).

Wang-Huang [19, loc. cit.] also proved the necessary conditions for the embeddings $B_{s_{1}}^{p, q} \hookrightarrow M^{p, q} \hookrightarrow B_{s_{2}}^{p, q}$. The necessity of (a) and (d) of Theorems 1.1 and 1.2 can be derived from the necessary condition of [19]. The necessity of (b) and (c) does not seem to follow from the latter, however. We shall give an independent proof for the necessity of our condition in this paper.

Our paper is organized as follows: In Section 2, we give the definitions and basic properties of modulation and local Hardy spaces. Sections 3 and 4 are devoted to the proofs of Theorems 1.1 and 1.2, respectively. 
2. Preliminaries. Let $\mathcal{S}\left(\mathbb{R}^{n}\right)$ and $\mathcal{S}^{\prime}\left(\mathbb{R}^{n}\right)$ be the Schwartz spaces of all rapidly decreasing smooth functions and tempered distributions, respectively. We define the Fourier transform $\mathcal{F} f$ and the inverse Fourier transform $\mathcal{F}^{-1} f$ of $f \in \mathcal{S}\left(\mathbb{R}^{n}\right)$ by

$$
\mathcal{F} f(\xi)=\widehat{f}(\xi)=\int_{\mathbb{R}^{n}} e^{-2 \pi i \xi \cdot x} f(x) d x \quad \text { and } \quad \mathcal{F}^{-1} f(x)=\int_{\mathbb{R}^{n}} e^{2 \pi i x \cdot \xi} f(\xi) d \xi .
$$

The notation $A \asymp B$ stands for $C^{-1} A \leq B \leq C A$ for some positive constant $C$.

We first recall the definition of modulation spaces. Let $0<p, q \leq \infty$, $s \in \mathbb{R}$, and let $\varphi \in \mathcal{S}\left(\mathbb{R}^{n}\right)$ be such that

$$
\operatorname{supp} \varphi \subset[-1,1]^{n} \text { and } \sum_{k \in \mathbb{Z}^{n}} \varphi(\xi-k)=1 \quad \text { for all } \xi \in \mathbb{R}^{n} .
$$

Then the modulation space $M_{s}^{p, q}\left(\mathbb{R}^{n}\right)$ consists of all $f \in \mathcal{S}^{\prime}\left(\mathbb{R}^{n}\right)$ such that

$$
\|f\|_{M_{s}^{p, q}}=\left(\sum_{k \in \mathbb{Z}^{n}}(1+|k|)^{s q}\|\varphi(D-k) f\|_{L^{p}}^{q}\right)^{1 / q}<\infty,
$$

where

$$
\varphi(D-k) f=\mathcal{F}^{-1}[\varphi(\cdot-k) \widehat{f}]=\left(M_{k} \Phi\right) * f,
$$

$\Phi(x)=\mathcal{F}^{-1} \varphi$ and $M_{k} \Phi(x)=e^{2 \pi i k \cdot x} \Phi(x)$. If $s=0$, we write $M^{p, q}\left(\mathbb{R}^{n}\right)$ instead of $M_{0}^{p, q}\left(\mathbb{R}^{n}\right)$. We remark that the definition of $M_{s}^{p, q}\left(\mathbb{R}^{n}\right)$ is independent of the choice of $\varphi, M_{s}^{p, q}\left(\mathbb{R}^{n}\right)$ is a quasi Banach space (Banach space if $1 \leq p, q \leq \infty)$, and $M_{s_{1}}^{p_{1}, q_{1}}\left(\mathbb{R}^{n}\right) \hookrightarrow M_{s_{2}}^{p_{2}, q_{2}}\left(\mathbb{R}^{n}\right)$ if $p_{1} \leq p_{2}, q_{1} \leq q_{2}$ and $s_{1} \geq s_{2}$. See Feichtinger [4], Gröchenig [8, Chapter 11], Kobayashi [10] and Triebel [17] for more details on modulation spaces.

We next recall the local Hardy spaces of Goldberg [6]. Let $0<p<\infty$, and let $\Psi \in \mathcal{S}\left(\mathbb{R}^{n}\right)$ be such that $\int_{\mathbb{R}^{n}} \Psi(x) d x \neq 0$. Then the local Hardy space $h^{p}\left(\mathbb{R}^{n}\right)$ consists of all $f \in \mathcal{S}^{\prime}\left(\mathbb{R}^{n}\right)$ such that

$$
\|f\|_{h^{p}}=\left\|\sup _{0<t<1}\left|\Psi_{t} * f\right|\right\|_{L^{p}}=\left\|\sup _{0<t<1}|\psi(t D) f|\right\|_{L^{p}}<\infty,
$$

where $\Psi_{t}(x)=t^{-n} \Psi(x / t)$ and $\psi=\widehat{\Psi}$. We remark that $h^{1}\left(\mathbb{R}^{n}\right) \hookrightarrow L^{1}\left(\mathbb{R}^{n}\right)([6$, Theorem 2]), $h^{p}\left(\mathbb{R}^{n}\right)=L^{p}\left(\mathbb{R}^{n}\right)$ if $1<p<\infty([6$, p. 30]), and the definition of $h^{p}\left(\mathbb{R}^{n}\right)$ is independent of the choice of $\Psi$ ([6, Theorem 1]). A function $a$ on $\mathbb{R}^{n}$ is said to be an $h^{p}$-atom of type $I$ if

$$
\begin{aligned}
& \operatorname{supp} a \subset Q \quad \text { with }|Q|<1, \quad\|a\|_{L^{\infty}} \leq|Q|^{-1 / p}, \\
& \int_{\mathbb{R}^{n}} x^{\alpha} a(x) d x=0 \quad \text { for all }|\alpha| \leq[n(1 / p-1)],
\end{aligned}
$$

where $Q$ is a cube (that is, $Q=x_{0}+[-r, r]^{n}$ for some $x_{0} \in \mathbb{R}^{n}$ and $r>0$ ), $|Q|$ is the Lebesgue measure of $Q$, and $[n(1 / p-1)]$ is the integer part of 
$n(1 / p-1)$. A function $a$ on $\mathbb{R}^{n}$ is also said to be an $h^{p}$-atom of type II if $\operatorname{supp} a \subset Q \quad$ with $|Q| \geq 1, \quad\|a\|_{L^{\infty}} \leq|Q|^{-1 / p}$.

We simply say that $a$ is an $h^{p}$-atom if it is an $h^{p}$-atom of type I or type II. Note that there exists a constant $C>0$ such that $\|a\|_{h^{p}} \leq C$ for all $h^{p}$-atoms $a\left(\left[6\right.\right.$, Lemma 5]). It is known that every $f \in h^{p}\left(\mathbb{R}^{n}\right)$ can be written as a sum of $h^{p}$-atoms:

$$
f=\sum_{i=1}^{\infty} \lambda_{i} a_{i} \quad \text { in } \mathcal{S}^{\prime}\left(\mathbb{R}^{n}\right),
$$

where $\left\{a_{i}\right\}$ is a collection of $h^{p}$-atoms and $\left\{\lambda_{i}\right\}$ is a sequence of complex numbers with $\sum_{i=1}^{\infty}\left|\lambda_{i}\right|^{p}<\infty$, and moreover,

$$
\|f\|_{h^{p}} \asymp \inf \left(\sum_{i=1}^{\infty}\left|\lambda_{i}\right|^{p}\right)^{1 / p}
$$

where the infimum is taken over all representations $f=\sum_{i=1}^{\infty} \lambda_{i} a_{i}([6$, Lemma 5]).

We end this section by quoting the following facts which will be used later on.

Lemma 2.1 ([11]). Let $0<p, q \leq \infty$ and $s \in \mathbb{R}$. If $\psi \in \mathcal{S}\left(\mathbb{R}^{n}\right)$ satisfies $|\psi| \geq c>0$ on $[-2,2]^{n}$, then

$$
\|f\|_{M_{s}^{p, q}} \asymp\left(\sum_{k \in \mathbb{Z}^{n}}(1+|k|)^{s q}\|\psi(D-k) f\|_{L^{p}}^{q}\right)^{1 / q} \quad \text { for all } f \in M_{s}^{p, q}\left(\mathbb{R}^{n}\right) .
$$

Lemma 2.2 ([20, Proposition 2.5]). Let $0<p, q_{1}, q_{2} \leq \infty$ and $s_{1}, s_{2} \in \mathbb{R}$. If $q_{1}>q_{2}$ and $s_{1}-s_{2}>n\left(1 / q_{2}-1 / q_{1}\right)$, then $M_{s_{1}}^{p, q_{1}}\left(\mathbb{R}^{n}\right) \hookrightarrow M_{s_{2}}^{p, q_{2}}\left(\mathbb{R}^{n}\right)$.

3. Proof of Theorem 1.1. The following proposition seems to be known to many people, but we give a proof for the reader's convenience. Then

Proposition 3.1. Let $0<p \leq 1$, and let $\varphi \in \mathcal{S}\left(\mathbb{R}^{n}\right)$ be as in (2.1).

$$
\|\varphi(D-k) f\|_{h^{p}} \asymp\|\varphi(D-k) f\|_{L^{p}}
$$

for all $f \in \mathcal{S}^{\prime}\left(\mathbb{R}^{n}\right)$ satisfying $\|\varphi(D-k) f\|_{h^{p}}<\infty$ or $\|\varphi(D-k) f\|_{L^{p}}<\infty$.

Proof. Let $\Psi \in \mathcal{S}$ with $\int_{\mathbb{R}^{n}} \Psi(x) d x=1$ and $\operatorname{supp} \Psi \subset\left\{x \in \mathbb{R}^{n}:|x| \leq 1\right\}$, and set $\psi=\widehat{\Psi}$. We first prove that $\|\varphi(D-k) f\|_{L^{p}} \leq C\|\varphi(D-k) f\|_{h^{p}}$. Since $\psi(t D) g=\Psi_{t} * g \rightarrow g$ in $\mathcal{S}^{\prime}$ as $t \rightarrow 0$ for all $g \in \mathcal{S}^{\prime}$, where $\Psi_{t}(x)=t^{-n} \Psi(x / t)$, we see that

$$
\begin{aligned}
& \psi(t D) \varphi(D-k) f(x)=\left(M_{k} \Phi\right) *(\psi(t D) f)(x) \\
& \quad=\left\langle\psi(t D) f,\left(M_{k} \Phi\right)(x-\cdot)\right\rangle_{\mathcal{S}^{\prime} \times \mathcal{S}} \rightarrow\left\langle f,\left(M_{k} \Phi\right)(x-\cdot)\right\rangle_{\mathcal{S}^{\prime} \times \mathcal{S}}=\varphi(D-k) f(x)
\end{aligned}
$$


as $t \rightarrow 0$ for all $x \in \mathbb{R}^{n}$. Hence,

$$
\|\varphi(D-k) f\|_{L^{p}} \leq\left\|\sup _{0<t<1}|\psi(t D)(\varphi(D-k) f)|\right\| L_{L^{p}}=\|\varphi(D-k) f\|_{h^{p}} .
$$

We next prove that $\|\varphi(D-k) f\|_{h^{p}} \leq C\|\varphi(D-k) f\|_{L^{p}}$. To do this, we recall that, for a compact subset $\Omega$ of $\mathbb{R}^{n}$ and $0<r<p$, there exists a constant $C>0$ such that

$$
\sup _{y \in \mathbb{R}^{n}} \frac{|f(x-y)|}{(1+|y|)^{n / r}} \leq C M\left(|f|^{r}\right)(x)^{1 / r}
$$

for all $f \in \mathcal{S}^{\prime}$ satisfying supp $\widehat{f} \subset \Omega$ and $x \in \mathbb{R}^{n}$, where $M$ is the HardyLittlewood maximal operator defined by

$$
M f(x)=\sup _{Q \ni x} \frac{1}{|Q|} \int_{Q}|f(y)| d y
$$

(see [18, Theorems 1.3.1 and 1.4.1]). Since

$$
\begin{aligned}
& \left|\Psi_{t} *(\varphi(D-k) f)(x)\right|=\left|\int_{\mathbb{R}^{n}} t^{-n} \Psi(y / t) \varphi(D-k) f(x-y) d y\right| \\
& \quad=\left|\int_{|y| \leq 1} \Psi(y) \varphi(D-k) f(x-t y) d y\right| \leq\|\Psi\|_{L^{1}} \sup _{|y| \leq 1}|\varphi(D-k) f(x-y)|
\end{aligned}
$$

for all $0<t<1$, we have

$$
\sup _{0<t<1}\left|\Psi_{t} *(\varphi(D-k) f)(x)\right| \leq C \sup _{|y| \leq 1}|\varphi(D-k) f(x-y)| .
$$

On the other hand, since supp $\mathcal{F}\left[M_{-k}(\varphi(D-k) f)\right] \subset[-1,1]^{n}$ for all $k \in \mathbb{Z}^{n}$, by (3.1) we have

$$
\begin{aligned}
\sup _{|y| \leq 1}|\varphi(D-k) f(x-y)| & \leq C \sup _{y \in \mathbb{R}^{n}} \frac{|\varphi(D-k) f(x-y)|}{(1+|y|)^{n / r}} \\
& =C \sup _{y \in \mathbb{R}^{n}} \frac{\left|\left[M_{-k}(\varphi(D-k) f)\right](x-y)\right|}{(1+|y|)^{n / r}} \\
& \leq C M\left(\left|M_{-k}(\varphi(D-k) f)\right|^{r}\right)(x)^{1 / r} \\
& =C M\left(|\varphi(D-k) f|^{r}\right)(x)^{1 / r} .
\end{aligned}
$$

Note that $p / r>1$ and the Hardy-Littlewood maximal operator $M$ is bounded on $L^{p / r}([1$, Theorem 2.5]). Therefore, it follows from (3.2) and (3.3) that

$$
\begin{aligned}
& \|\varphi(D-k) f\|_{h^{p}}=\left\|\sup _{0<t<1}\left|\Psi_{t} *(\varphi(D-k) f)\right|\right\|_{L^{p}} \\
& \quad \leq C\left\|M\left(|\varphi(D-k) f|^{r}\right)(x)^{1 / r}\right\| L_{L^{p}}=C\left\|M\left(|\varphi(D-k) f|^{r}\right)(x)\right\|_{L^{p / r}}^{1 / r} \\
& \quad \leq\left. C\|\| \varphi(D-k) f\right|^{r}\left\|_{L^{p / r}}^{1 / r}=C\right\| \varphi(D-k) f \|_{L^{p}} \text {. }
\end{aligned}
$$


Lemma 3.2. Let $0<p \leq 1,0<q \leq \infty$ and $s \in \mathbb{R}$. If $M_{s}^{p, q}\left(\mathbb{R}^{n}\right) \hookrightarrow$ $h^{p}\left(\mathbb{R}^{n}\right)$, then there exists a constant $C>0$ such that

$$
\left(\sum_{k \in \mathbb{Z}^{n}}\left|c_{k}\right|^{p}\right)^{1 / p} \leq C\left(\sum_{k \in \mathbb{Z}^{n}}(1+|k|)^{s q}\left|c_{k}\right|^{q}\right)^{1 / q}
$$

for all finitely supported sequences $\left\{c_{k}\right\}_{k \in \mathbb{Z}^{n}}$ (that is, $c_{k}=0$ except for a finite number of $k$ 's).

Proof. Let $\eta \in \mathcal{S} \backslash\{0\}$ be such that $\operatorname{supp} \eta \subset[-1 / 2,1 / 2]^{n}$. For a finitely supported sequence $\left\{c_{l}\right\}_{l \in \mathbb{Z}^{n}}$, we set

$$
f(x)=\sum_{l \in \mathbb{Z}^{n}} c_{l} e^{2 \pi i l \cdot x} \eta(x-l) .
$$

Let $\varphi \in \mathcal{S}$ be as in (2.1). Since

$$
\widehat{f}(\xi)=\sum_{l \in \mathbb{Z}^{n}} c_{l} e^{2 \pi i|l|^{2}} e^{-2 \pi i l \cdot \xi} \widehat{\eta}(\xi-l),
$$

we see that

$$
\varphi(D-k) f(x)=\sum_{l \in \mathbb{Z}^{n}} c_{l} e^{2 \pi i|l|^{2}} \int_{\mathbb{R}^{n}} e^{2 \pi i(x-l) \cdot \xi} \varphi(\xi-k) \widehat{\eta}(\xi-l) d \xi .
$$

Using

$$
\int_{\mathbb{R}^{n}}(1+|x-y|)^{-M}(1+|y|)^{-M} d y \leq C(1+|x|)^{-M},
$$

where $M>n$, and

$$
\begin{aligned}
(x-l)^{\alpha} & \int_{\mathbb{R}^{n}} e^{2 \pi i(x-l) \cdot \xi} \varphi(\xi-k) \widehat{\eta}(\xi-l) d \xi \\
\quad= & \sum_{\alpha_{1}+\alpha_{2}=\alpha} C_{\alpha_{1}, \alpha_{2}} \int_{\mathbb{R}^{n}} e^{2 \pi i(x-l) \cdot \xi}\left(\partial^{\alpha_{1}} \varphi\right)(\xi-k)\left(\partial^{\alpha_{2}} \widehat{\eta}\right)(\xi-l) d \xi,
\end{aligned}
$$

we have

$$
\left|\int_{\mathbb{R}^{n}} e^{2 \pi i(x-l) \cdot \xi} \varphi(\xi-k) \widehat{\eta}(\xi-l) d \xi\right| \leq C_{N}(1+|x-l|)^{-N}(1+|k-l|)^{-N}
$$

for all $N \geq 1$. Let $N$ be an integer satisfying $N>\max \{n / p+|s|, n / q+|s|\}$. Then, by (3.4) and (3.5),

$$
|\varphi(D-k) f(x)| \leq C \sum_{l \in \mathbb{Z}^{n}} \frac{\left|c_{l}\right|}{(1+|x-l|)^{N}(1+|k-l|)^{N}},
$$


which yields

$$
\begin{aligned}
\|\varphi(D-k) f\|_{L^{p}}^{p} & \leq C \sum_{l \in \mathbb{Z}^{n}} \frac{\left|c_{l}\right|^{p}}{(1+|k-l|)^{N p}}\left\|(1+|\cdot-l|)^{-N}\right\|_{L^{p}}^{p} \\
& =C \sum_{l \in \mathbb{Z}^{n}} \frac{\left|c_{l}\right|^{p}}{(1+|k-l|)^{N p}} .
\end{aligned}
$$

Hence, since

$$
\begin{aligned}
\|f\|_{M_{s}^{p, q}} & =\left\{\sum_{k \in \mathbb{Z}^{n}}(1+|k|)^{s q}\|\varphi(D-k) f\|_{L^{p}}^{q}\right\}^{1 / q} \\
& =\left\{\sum_{k \in \mathbb{Z}^{n}}\left((1+|k|)^{s p}\|\varphi(D-k) f\|_{L^{p}}^{p}\right)^{q / p}\right\}^{1 / q} \\
& \leq C\left\{\sum_{k \in \mathbb{Z}^{n}}\left((1+|k-l|)^{|s| p}(1+|l|)^{s p} \sum_{l \in \mathbb{Z}^{n}} \frac{\left|c_{l}\right|^{p}}{(1+|k-l|)^{N p}}\right)^{q / p}\right\}^{1 / q} \\
& =C\left\{\sum_{k \in \mathbb{Z}^{n}}\left(\sum_{l \in \mathbb{Z}^{n}} \frac{(1+|l|)^{s p}\left|c_{l}\right|^{p}}{(1+|k-l|)^{(N-|s|) p}}\right)^{q / p}\right\}^{1 / q},
\end{aligned}
$$

Young's inequality if $q / p \geq 1$ and Fubini's theorem if $q / p<1$ yield (3.6)

$$
\begin{aligned}
& \|f\|_{M_{s}^{p, q}} \leq C \begin{cases}\left\{\sum_{k \in \mathbb{Z}^{n}}\left(\sum_{l \in \mathbb{Z}^{n}} \frac{(1+|l|)^{s p}\left|c_{l}\right|^{p}}{(1+|k-l|)^{(N-|s|) p}}\right)^{q / p}\right\}^{1 / q} & \text { if } q / p \geq 1 \\
\left\{\sum_{k \in \mathbb{Z}^{n}} \sum_{l \in \mathbb{Z}^{n}} \frac{(1+|l|)^{s q}\left|c_{l}\right|^{q}}{(1+|k-l|)^{(N-|s|) q}}\right\}^{1 / q} & \text { if } q / p<1\end{cases} \\
& \leq C \begin{cases}\left\|\left\{(1+|k|)^{-(N-|s|) p}\right\}\right\|_{\ell^{1}}^{1 / p}\left\|\left\{(1+|l|)^{s} c_{l}\right\}\right\|_{\ell^{q}} & \text { if } q / p \geq 1 \\
\left\|\left\{(1+|k|)^{-(N-|s|) q}\right\}\right\|_{\ell^{1}}^{1 / q}\left\|\left\{(1+|l|)^{s} c_{l}\right\}\right\|_{\ell^{q}} & \text { if } q / p<1\end{cases} \\
& \leq C\left\|\left\{(1+|l|)^{s} c_{l}\right\}\right\|_{\ell^{q}} .
\end{aligned}
$$

On the other hand, as $f \in \mathcal{S}$, we have $f(x)=\lim _{t \rightarrow 0} \psi(t D) f(x)$ for all $x \in \mathbb{R}^{n}$, where $\psi \in \mathcal{S}$ satisfies $\psi(0)=1$. This implies

$$
\|f\|_{L^{p}} \leq\left\|\sup _{0<t<1}|\psi(t D) f|\right\|_{L^{p}}=\|f\|_{h^{p}} .
$$

Since $\operatorname{supp} \eta(\cdot-l) \subset l+[-1 / 2,1 / 2]^{n}$ for all $l \in \mathbb{Z}^{n}$, we see that

$$
\begin{aligned}
\|f\|_{L^{p}} & =\left(\int_{\mathbb{R}^{n}}\left|\sum_{l \in \mathbb{Z}^{n}} c_{l} e^{2 \pi i l \cdot x} \eta(x-l)\right|^{p} d x\right)^{1 / p} \\
& =\left(\int_{\mathbb{R}^{n}} \sum_{l \in \mathbb{Z}^{n}}\left|c_{l} e^{2 \pi i l \cdot x} \eta(x-l)\right|^{p} d x\right)^{1 / p}=\|\eta\|_{L^{p}}\left\|\left\{c_{l}\right\}\right\|_{\ell^{p}} .
\end{aligned}
$$


By our assumption $M_{s}^{p, q} \hookrightarrow h^{p}$ and (3.6)-(3.8), we obtain

$$
C_{1}\left\|\left\{c_{l}\right\}\right\|_{\ell^{p}} \leq\|f\|_{h^{p}} \leq C_{2}\|f\|_{M_{s}^{p, q}} \leq C_{3}\left\|\left\{(1+|l|)^{s} c_{l}\right\}\right\|_{\ell^{q}} .
$$

We are now ready to prove Theorem 1.1.

Proof of Theorem 1.1. We first prove that $M^{p, p} \hookrightarrow h^{p}$. Let $\varphi \in \mathcal{S}$ be as in (2.1). By Proposition 3.1,

$$
\begin{aligned}
\|f\|_{h^{p}}^{p} & =\left\|\sum_{k \in \mathbb{Z}^{n}} \varphi(D-k) f\right\|_{h^{p}}^{p} \leq \sum_{k \in \mathbb{Z}^{n}}\|\varphi(D-k) f\|_{h^{p}}^{p} \\
& \leq C \sum_{k \in \mathbb{Z}^{n}}\|\varphi(D-k) f\|_{L^{p}}^{p}=\|f\|_{M^{p, p}}^{p},
\end{aligned}
$$

that is, $M^{p, p} \hookrightarrow h^{p}$. On the other hand, if $p \geq q$ and $s \geq 0$, or if $p<q$ and $s>n(1 / p-1 / q)$, then $M_{s}^{p, q} \hookrightarrow M^{p, p}$, where we have used Lemma 2.2 in the case $p<q$ and $s>n(1 / p-1 / q)$. Hence, condition (a) or (b) gives $M_{s}^{p, q} \hookrightarrow h^{p}$.

We next prove that the embedding $M_{s}^{p, q} \hookrightarrow h^{p}$ implies (a) or (b). Assume that $M_{s}^{p, q} \hookrightarrow h^{p}$. Then, from Lemma 3.2,

$$
\left(\sum_{k \in \mathbb{Z}^{n}}\left|c_{k}\right|^{p}\right)^{1 / p} \leq C\left(\sum_{k \in \mathbb{Z}^{n}}(1+|k|)^{s q}\left|c_{k}\right|^{q}\right)^{1 / q}
$$

for all finitely supported sequences $\left\{c_{k}\right\}_{k \in \mathbb{Z}^{n}}$. Setting $c_{k}=(1+|k|)^{-s}\left|d_{k}\right|^{1 / p}$, we see that (3.9) is equivalent to

$$
\sum_{k \in \mathbb{Z}^{n}}(1+|k|)^{-s p}\left|d_{k}\right| \leq C\left(\sum_{k \in \mathbb{Z}^{n}}\left|d_{k}\right|^{q / p}\right)^{p / q}
$$

for all finitely supported sequences $\left\{d_{k}\right\}_{k \in \mathbb{Z}^{n}}$. If we choose the sequence $\left\{d_{k}\right\}$ defined by $d_{k}=1$ when $k=(N, 0, \ldots, 0)$ and $d_{k}=0$ when $k \neq(N, 0, \ldots, 0)$, where $N$ is a positive integer, then (3.10) implies $(1+N)^{-s p} \leq C$ for all $N \geq 1$. This means that necessarily $s \geq 0$.

In the case $p \geq q$, we have nothing to prove any more, that is, we have condition (a). Assume that $p<q$. In the case $p<q<\infty$, since $1<q / p<\infty$, by (3.10) we have

$$
\left\|\left\{(1+|k|)^{-s p}\right\}\right\|_{\ell^{(q / p)^{\prime}}}=\sup \left|\sum_{k \in \mathbb{Z}^{n}}(1+|k|)^{-s p} d_{k}\right| \leq C,
$$

where the supremum is taken over all finitely supported sequences $\left\{d_{k}\right\}_{k \in \mathbb{Z}^{n}}$ such that $\left\|\left\{d_{k}\right\}\right\|_{\ell^{q} / p}=1$. In the same way, we can prove $\left\|\left\{(1+|k|)^{-s p}\right\}\right\|_{\ell^{1}}$ $\leq C$ when $q=\infty$. Hence, $p, q, s$ must satisfy $s p(q / p)^{\prime}>n$, that is, $s>$ $n(1 / p-1 / q)$. Therefore, we have condition (b). 


\section{Proof of Theorem 1.2}

Lemma 4.1. Let $0<p \leq 1$ and $0<q \leq 2$. Then there exists a constant $C>0$ such that

$$
\|a\|_{M_{-n(1 / p+1 / q-1)}^{p, q}} \leq C \quad \text { for all } h^{p} \text {-atoms a. }
$$

Proof. Let $\Psi \in \mathcal{S}$ be such that $\operatorname{supp} \Psi \subset[-1,1]^{n}$ and $|\widehat{\Psi}| \geq c>0$ on $[-2,2]^{n}$ (see the proof of Lemma 4.3 for the existence of such a function), and set $\psi=\widehat{\Psi}$ and $s=-n(1 / p+1 / q-1)$. In order to prove Lemma 4.1, we shall prove that there exists a constant $C>0$ such that

$$
\left(\sum_{k \in \mathbb{Z}^{n}}(1+|k|)^{s q}\|\psi(D-k) a\|_{L^{p}}^{q}\right)^{1 / q} \leq C \quad \text { for all } h^{p} \text {-atoms } a .
$$

Before proving (4.1), we shall see that (4.1) implies Lemma 4.1. If $a$ is an $h^{p}$-atom of type I (resp. type II) and $\varepsilon>0$ is sufficiently small, then $\left(\varrho_{\varepsilon} * a\right) /\left(2^{n}\|\varrho\|_{L^{1}}\right)$ is also an $h^{p}$-atom of type I (resp. type II), where $\varrho \in$ $\mathcal{S}$ satisfies $\int_{\mathbb{R}^{n}} \varrho(x) d x=1$ and supp $\varrho$ is compact, and we have used the notation $\varrho_{\varepsilon}(x)=\varepsilon^{-n} \varrho(x / \varepsilon)$. Thus, (4.1) gives

$$
\left(\sum_{k \in \mathbb{Z}^{n}}(1+|k|)^{s q}\left\|\psi(D-k)\left(\varrho_{\varepsilon} * a\right)\right\|_{L^{p}}^{q}\right)^{1 / q} \leq C
$$

for all sufficiently small $\varepsilon>0$. Let $\varphi \in \mathcal{S}$ be as in (2.1). Since $|\psi| \geq c>0$ on $[-2,2]^{n}$ and $\varrho_{\varepsilon} * a \in \mathcal{S} \subset M_{s}^{p, q}$ for all $\varepsilon>0$, by Lemma 2.1 we have

$$
\begin{aligned}
\left\|\varrho_{\varepsilon} * a\right\|_{M_{s}^{p, q}} & =\left(\sum_{k \in \mathbb{Z}^{n}}(1+|k|)^{s q}\left\|\varphi(D-k)\left(\varrho_{\varepsilon} * a\right)\right\|_{L^{p}}^{q}\right)^{1 / q} \\
& \asymp\left(\sum_{k \in \mathbb{Z}^{n}}(1+|k|)^{s q}\left\|\psi(D-k)\left(\varrho_{\varepsilon} * a\right)\right\|_{L^{p}}^{q}\right)^{1 / q}
\end{aligned}
$$

for all $\varepsilon>0$. Note that $\varrho_{\varepsilon} * a \rightarrow a$ in $\mathcal{S}^{\prime}$ as $\varepsilon \rightarrow 0$, and consequently $\varphi(D-k)\left(\varrho_{\varepsilon} * a\right)(x) \rightarrow \varphi(D-k) a(x)$ as $\varepsilon \rightarrow 0$ for all $x \in \mathbb{R}^{n}$. Then, by (4.2), (4.3) and Fatou's lemma, we see that

$$
\begin{aligned}
\|a\|_{M_{s}^{p, q}} & =\left(\sum_{k \in \mathbb{Z}^{n}}(1+|k|)^{s q}\left\|\lim _{\varepsilon \rightarrow 0} \varphi(D-k)\left(\varrho_{\varepsilon} * a\right)\right\|_{L^{p}}^{q}\right)^{1 / q} \\
& \leq \liminf _{\varepsilon \rightarrow 0}\left(\sum_{k \in \mathbb{Z}^{n}}(1+|k|)^{s q}\left\|\varphi(D-k)\left(\varrho_{\varepsilon} * a\right)\right\|_{L^{p}}^{q}\right)^{1 / q} \\
& \leq C \liminf _{\varepsilon \rightarrow 0}\left(\sum_{k \in \mathbb{Z}^{n}}(1+|k|)^{s q}\left\|\psi(D-k)\left(\varrho_{\varepsilon} * a\right)\right\|_{L^{p}}^{q}\right)^{1 / q} \leq C,
\end{aligned}
$$

and this is the desired result.

Let us prove (4.1). By the translation invariance of the quasi-norm $\|\cdot\|_{L^{p}}$, we may assume that $a$ is an $h^{p}$-atom with $Q=[-r, r]^{n}$. If $a$ is an $h^{p}$-atom 
with $Q=[-r, r]^{n}$, then

$$
\operatorname{supp}\left(M_{k} \Psi\right) * a \subset[-1,1]^{n}+[-r, r]^{n}=[-(1+r), 1+r]^{n},
$$

and Hölder's inequality gives

$$
\begin{aligned}
\left\|\left(M_{k} \Psi\right) * a\right\|_{L^{p}} & \leq\left|[-(1+r), 1+r]^{n}\right|^{1 / p-1 / 2}\left\|\left(M_{k} \Psi\right) * a\right\|_{L^{2}} \\
& \leq C\left\|\left(M_{k} \Psi\right) * a\right\|_{L^{2}} \begin{cases}1 & \text { if } r<1 / 2, \\
|Q|^{1 / p-1 / 2} & \text { if } r \geq 1 / 2 .\end{cases}
\end{aligned}
$$

We first consider the case that $a$ is an $h^{p}$-atom of type I with $Q=[-r, r]^{n}$, and note that $r<1 / 2$. We split

$$
\begin{aligned}
& \sum_{k \in \mathbb{Z}^{n}}(1+|k|)^{-n(1 / p+1 / q-1) q}\|\psi(D-k) a\|_{L^{p}}^{q} \\
& \quad=\left(\sum_{|k| \leq|Q|^{-1 / n}}+\sum_{|k|>|Q|^{-1 / n}}\right)(1+|k|)^{-n(1 / p+1 / q-1) q}\left\|\left(M_{k} \Psi\right) * a\right\|_{L^{p}}^{q} .
\end{aligned}
$$

We write $N=[n(1 / p-1)]$. By (2.3) and Taylor's formula,

$$
\begin{aligned}
& \left(M_{k} \Psi\right) * a(x)=\int_{\mathbb{R}^{n}}\left(M_{k} \Psi(x-y)-\sum_{|\alpha| \leq N} \frac{\partial^{\alpha} M_{k} \Psi(x)}{\alpha !}(-y)^{\alpha}\right) a(y) d y \\
& =\int_{|y| \leq \sqrt{n} r}\left((N+1) \sum_{|\alpha|=N+1} \frac{(-y)^{\alpha}}{\alpha !} \int_{0}^{1}(1-t)^{N}\left(\partial^{\alpha} M_{k} \Psi\right)(x-t y) d t\right) a(y) d y .
\end{aligned}
$$

Since $\left\|\partial^{\alpha} M_{k} \Psi\right\|_{L^{\infty}} \leq C_{\alpha}(1+|k|)^{|\alpha|}$ for all $k \in \mathbb{Z}^{n}$, by (2.2) we have

$$
\left\|\left(M_{k} \Psi\right) * a\right\|_{L^{\infty}} \leq C(1+|k|)^{N+1} r^{N+n+1-n / p} .
$$

Hence, from (4.4),

$$
\begin{aligned}
\left\|\left(M_{k} \Psi\right) * a\right\|_{L^{p}} & \leq C(1+|k|)^{N+1} r^{N+n+1-n / p}\left|[-(1+r), 1+r]^{n}\right|^{1 / p} \\
& \leq C(1+|k|)^{N+1}|Q|^{(N+1) / n-(1 / p-1)} .
\end{aligned}
$$

This implies

$$
\begin{aligned}
& \sum_{|k| \leq|Q|^{-1 / n}}(1+|k|)^{-n(1 / p+1 / q-1) q}\left\|\left(M_{k} \Psi\right) * a\right\|_{L^{p}}^{q} \\
& \leq C|Q|^{\{(N+1) / n-(1 / p-1)\} q} \sum_{|k| \leq|Q|^{-1 / n}}(1+|k|)^{\{N+1-n(1 / p-1)\} q-n} \leq C,
\end{aligned}
$$

where we have used the fact that $\{N+1-n(1 / p-1)\} q>0$. On the other hand, since $a \in L^{2}$ and $M^{2,2}=L^{2}$, by Lemma 2.1, (2.2) and (4.5) we have

$$
\begin{aligned}
\left(\sum_{k \in \mathbb{Z}^{n}}\left\|\left(M_{k} \Psi\right) * a\right\|_{L^{p}}^{2}\right)^{1 / 2} & \leq C\left(\sum_{k \in \mathbb{Z}^{n}}\left\|\left(M_{k} \Psi\right) * a\right\|_{L^{2}}^{2}\right)^{1 / 2} \\
& \leq C\|a\|_{L^{2}} \leq C|Q|^{1 / 2-1 / p}
\end{aligned}
$$


which yields

$$
\begin{aligned}
& \sum_{|k|>|Q|^{-1 / n}}(1+|k|)^{-n(1 / p+1 / q-1) q}\left\|\left(M_{k} \Psi\right) * a\right\|_{L^{p}}^{q} \\
\leq & \left(\sum_{|k|>|Q|^{-1 / n}}(1+|k|)^{-n(1 / p+1 / q-1) q u}\right)^{1 / u}\left(\sum_{|k|>|Q|^{-1 / n}}\left\|\left(M_{k} \Psi\right) * a\right\|_{L^{p}}^{2}\right)^{q / 2} \\
\leq & C|Q|^{(1 / p+1 / q-1) q-1 / u}|Q|^{(1 / 2-1 / p) q}=C|Q|^{0}=C,
\end{aligned}
$$

where $1 /(2 / q)+1 / u=1$ and where we have used the fact that $n(1 / p+1 / q$ $-1) q u>n$. Combining (4.7) and (4.8), we obtain (4.1) in the case that $a$ is an $h^{p}$-atom of type I with $Q=[-r, r]^{n}$.

We next consider the case where $a$ is an $h^{p}$-atom of type II with $Q=$ $[-r, r]^{n}$, and note that $r \geq 1 / 2$. In the same way as in (4.8), by (2.4) and (4.5) we have

$$
\begin{aligned}
& \sum_{k \in \mathbb{Z}^{n}}(1+|k|)^{-n(1 / p+1 / q-1) q}\left\|\left(M_{k} \Psi\right) * a\right\|_{L^{p}}^{q} \\
& \leq C|Q|^{(1 / p-1 / 2) q} \sum_{k \in \mathbb{Z}^{n}}(1+|k|)^{-n(1 / p+1 / q-1) q}\left\|\left(M_{k} \Psi\right) * a\right\|_{L^{2}}^{q} \\
& \leq C|Q|^{(1 / p-1 / 2) q}\left(\sum_{k \in \mathbb{Z}^{n}}(1+|k|)^{-n(1 / p+1 / q-1) q u}\right)^{1 / u}\left(\sum_{k \in \mathbb{Z}^{n}}\left\|\left(M_{k} \Psi\right) * a\right\|_{L^{2}}^{2}\right)^{q / 2} \\
& \leq C|Q|^{(1 / p-1 / 2) q}\|a\|_{L^{2}}^{q} \leq C|Q|^{(1 / p-1 / 2) q}|Q|^{(1 / 2-1 / p) q}=C|Q|^{0}=C .
\end{aligned}
$$

Lemma 4.2. Let $0<p \leq 1$. Then there exists a constant $C>0$ such that

$$
\|a\|_{M_{-n(1 / p-1)}^{p, \infty}} \leq C \quad \text { for all } h^{p} \text {-atoms } a .
$$

Proof. By the same reason as in the proof of Lemma 4.1, it is enough to prove that there exists a constant $C>0$ such that

$$
\sup _{k \in \mathbb{Z}^{n}}(1+|k|)^{-n(1 / p-1)}\left\|\left(M_{k} \Psi\right) * a\right\|_{L^{p}} \leq C
$$

for all $h^{p}$-atoms $a$, where $\Psi \in \mathcal{S}$ is as in the proof of Lemma 4.1, and we may assume that the cube $Q$ corresponding to the atom $a$ is centered at the origin.

We first consider the case where $a$ is an $h^{p}$-atom of type I with $Q=$ $[-r, r]^{n}$, and note that $r<1 / 2$. By (2.2), (4.4) and Hölder's inequality,

$$
\left\|\left(M_{k} \Psi\right) * a\right\|_{L^{p}} \leq C\left\|\left(M_{k} \Psi\right) * a\right\|_{L^{1}} \leq C\left\|M_{k} \Psi\right\|_{L^{1}}\|a\|_{L^{1}} \leq C|Q|^{1-1 / p} .
$$

We write $N=[n(1 / p-1)]$, and note that $N+1-n(1 / p-1)>0$. Then it 
follows from (4.6) and (4.9) that

$$
\begin{aligned}
(1 & +|k|)^{-n(1 / p-1)}\left\|\left(M_{k} \Psi\right) * a\right\|_{L^{p}} \\
& \leq C \begin{cases}(1+|k|)^{N+1-n(1 / p-1)}|Q|^{(N+1) / n-(1 / p-1)} & \text { if }|k| \leq|Q|^{-1 / n} \\
(1+|k|)^{-n(1 / p-1)}|Q|^{1-1 / p} & \text { if }|k|>|Q|^{-1 / n}\end{cases} \\
& \leq C \begin{cases}|Q|^{-\{(N+1) / n-(1 / p-1)\}}|Q|^{(N+1) / n-(1 / p-1)} & \text { if }|k| \leq|Q|^{-1 / n} \\
|Q|^{1 / p-1}|Q|^{1-1 / p} & \text { if }|k|>|Q|^{-1 / n}\end{cases}
\end{aligned}
$$

which implies

$$
\sup _{k \in \mathbb{Z}^{n}}(1+|k|)^{-n(1 / p-1)}\left\|\left(M_{k} \Psi\right) * a\right\|_{L^{p}} \leq C .
$$

We next consider the case where $a$ is an $h^{p}$-atom of type II with $Q=$ $[-r, r]^{n}$, and note that $r \geq 1 / 2$. From (2.4) and (4.5),

$$
\left\|\left(M_{k} \Psi\right) * a\right\|_{L^{p}} \leq C|Q|^{1 / p-1 / 2}\left\|\left(M_{k} \Psi\right) * a\right\|_{L^{2}} \leq C|Q|^{1 / p-1 / 2}\|a\|_{L^{2}} \leq C .
$$

Hence,

$$
\sup _{k \in \mathbb{Z}^{n}}(1+|k|)^{-n(1 / p-1)}\left\|\left(M_{k} \Psi\right) * a\right\|_{L^{p}} \leq C \sup _{k \in \mathbb{Z}^{n}}(1+|k|)^{-n(1 / p-1)} \leq C .
$$

Lemma 4.3. Let $\varepsilon>0$, and let $N$ be a non-negative integer. Then there exists a function $a \in \mathcal{S}\left(\mathbb{R}^{n}\right)$ such that

$$
\begin{aligned}
& \operatorname{supp} a \subset[-\varepsilon, \varepsilon]^{n}, \quad\|a\|_{L^{\infty}} \leq 1, \\
& \int_{\mathbb{R}^{n}} x^{\alpha} a(x) d x=0 \quad \text { for all }|\alpha| \leq N, \\
& |\widehat{a}(\xi)| \geq c>0 \quad \text { for } \text { all } 1 / 2 \leq|\xi| \leq 2 .
\end{aligned}
$$

Proof. Our proof is based on the argument of [5, Theorem 2.6]. Let $\varphi \in \mathcal{S}$ be such that $\operatorname{supp} \varphi \subset[-1,1]^{n}$ and $\widehat{\varphi}(0) \neq 0$. We may assume that $\varepsilon>0$ is sufficiently small. For $\varepsilon>0$, we set $\varphi_{\varepsilon}(x)=\varepsilon^{-n} \varphi(x / \varepsilon)$, and note that $\operatorname{supp} \varphi_{\varepsilon} \subset[-\varepsilon, \varepsilon]^{n}$. Since $\widehat{\varphi_{\varepsilon}}(\xi)=\widehat{\varphi}(\varepsilon \xi)$ and there exists $\delta>0$ such that $|\widehat{\varphi}(\xi)| \geq|\widehat{\varphi}(0)| / 2$ for all $|\xi| \leq \delta$, we see that if $\varepsilon<\delta / 2$ then $\left|\widehat{\varphi_{\varepsilon}}(\xi)\right| \geq|\widehat{\varphi}(0)| / 2>0$ for all $|\xi| \leq 2$. Hence, there exists $\psi \in \mathcal{S}$ such that $\operatorname{supp} \psi \subset[-\varepsilon, \varepsilon]^{n}$ and $|\widehat{\psi}(\xi)| \geq c>0$ for all $|\xi| \leq 2$. For such a function $\psi \in \mathcal{S}$ and a positive integer $M$ satisfying $M>N / 2$, we set $\eta=(-\Delta)^{M} \psi$, where $\Delta=\sum_{j=1}^{n} \partial^{2} / \partial x_{j}^{2}$. Then we can check that $\eta \in \mathcal{S}$ satisfies supp $\eta \subset$ $[-\varepsilon, \varepsilon]^{n},|\widehat{\eta}(\xi)| \geq c>0$ for all $1 / 2 \leq|\xi| \leq 2$ and $\int_{\mathbb{R}^{n}} x^{\alpha} \eta(x) d x=0$ for all $|\alpha| \leq N$. Therefore, $a=\eta /\|\eta\|_{L^{\infty}}$ satisfies the desired conditions.

In the proof of the following lemma, we use the fact that there exists $\Psi \in \mathcal{S}\left(\mathbb{R}^{n}\right)$ such that

$$
\begin{gathered}
\Psi=1 \quad \text { on }[-\delta / 4, \delta / 4]^{n}, \quad \operatorname{supp} \Psi \subset[-3 \delta / 8,3 \delta / 8]^{n}, \\
|\widehat{\Psi}| \geq c>0 \quad \text { on }[-2,2]^{n},
\end{gathered}
$$


where $0<\delta<1$ is a sufficiently small number. In fact, we can take $\Phi \in$ $\mathcal{S}\left(\mathbb{R}^{n}\right)$ such that $\Phi=1$ on $[-1 / 4,1 / 4]^{n}, \operatorname{supp} \Phi \subset[-3 / 8,3 / 8]^{n}$ and $\widehat{\Phi}(0) \neq 0$. As in the proof of Lemma 4.3, we can check that $\Phi(x / \delta)$ satisfies (4.10) if $\delta>0$ is sufficiently small.

Lemma 4.4. Let $0<p \leq 1,0<q \leq \infty$ and $s \in \mathbb{R}$. If $h^{p}\left(\mathbb{R}^{n}\right) \hookrightarrow$ $M_{s}^{p, q}\left(\mathbb{R}^{n}\right)$, then there exists a constant $C>0$ such that

$$
\left\{\sum_{k \neq 0}|k|^{(n(1 / p-1)+s) q}\left(\sum_{|k| / 2 \leq|l| \leq 2|k|}\left|c_{\ell}\right|^{p}\right)^{q / p}\right\}^{1 / q} \leq C\left(\sum_{k \neq 0}\left|c_{k}\right|^{p}\right)^{1 / p}
$$

for all finitely supported sequences $\left\{c_{k}\right\}_{k \in \mathbb{Z}^{n} \backslash\{0\}}$.

Proof. Let $\Psi \in \mathcal{S}$ be as in (4.10) with $0<\delta<1$, and let $a \in \mathcal{S}$ be as in Lemma 4.3 with $\varepsilon=\delta / 8$ and $N=[n(1 / p-1)]$. For a finitely supported sequence $\left\{c_{l}\right\}_{l \in \mathbb{Z}^{n} \backslash\{0\}}$, we set

$$
f(x)=\sum_{l \neq 0} c_{l}|l|^{n / p} a(|l|(x-l)) .
$$

Note that

$$
\begin{aligned}
& \operatorname{supp}|l|^{n / p} a(|l|(\cdot-l)) \subset l+[-\delta /(8|l|), \delta /(8|l|)]^{n}, \\
& \left\||l|^{n / p} a(|l|(\cdot-l))\right\|_{L^{\infty}} \leq(\delta / 4)^{n / p}\left|l+[-\delta /(8|l|), \delta /(8|l|)]^{n}\right|^{-1 / p}, \\
& \int_{\mathbb{R}^{n}} x^{\alpha}|l|^{n / p} a(|l|(x-l)) d x=0 \quad \text { for all }|\alpha| \leq[n(1 / p-1)],
\end{aligned}
$$

and these mean that $(\delta / 4)^{-n / p}|l|^{n / p} a(|l|(\cdot-l))$ is an $h^{p}$-atom of type I for all $l \neq 0$. Hence,

$$
\|f\|_{h^{p}}^{p} \leq \sum_{l \neq 0}\left|c_{l}\right|^{p}\left\||l|^{n / p} a(|l|(\cdot-l))\right\|_{h^{p}}^{p} \leq C \sum_{l \neq 0}\left|c_{l}\right|^{p} .
$$

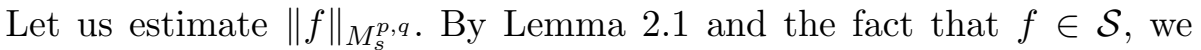
have

$$
\|f\|_{M_{s}^{p, q}} \asymp\left(\sum_{k \in \mathbb{Z}^{n}}(1+|k|)^{s q}\left\|\left(M_{k} \Psi\right) * f\right\|_{L^{p}}^{q}\right)^{1 / q} .
$$

Since $0<\delta<1$,

$$
\operatorname{supp} a(|l|(\cdot-l)) \subset l+[-\delta /(8|l|), \delta /(8|l|)]^{n} \subset l+[-\delta / 8, \delta / 8]^{n}
$$

for all $l \neq 0$ and

$$
\operatorname{supp} \Psi(x-\cdot) \subset x+[-3 \delta / 8,3 \delta / 8]^{n} \subset m+[-\delta / 2, \delta / 2]^{n}
$$

for all $x \in m+[-\delta / 8, \delta / 8]^{n}$ and $m \in \mathbb{Z}^{n}$, we see that 


$$
\begin{aligned}
& \left\|\left(M_{k} \Psi\right) * f\right\|_{L^{p}}^{p} \geq \sum_{m \in \mathbb{Z}^{n}} \int_{m+[-\delta / 8, \delta / 8]^{n}}\left|\left(M_{k} \Psi\right) * f(x)\right|^{p} d x \\
& =\left.\left.\sum_{m \in \mathbb{Z}^{n}} \int_{m+[-\delta / 8, \delta / 8]^{n}}\left|\int_{\mathbb{R}^{n}} e^{2 \pi i k \cdot(x-y)} \Psi(x-y) \sum_{l \neq 0} c_{l}\right| l\right|^{n / p} a(|l|(y-l)) d y\right|^{p} d x \\
& =\left.\left.\sum_{m \neq 0} \int_{m+[-\delta / 8, \delta / 8]^{n}}\left|\int_{\mathbb{R}^{n}} e^{-2 \pi i k \cdot y} \Psi(x-y) c_{m}\right| m\right|^{n / p} a(|m|(y-m)) d y\right|^{p} d x
\end{aligned}
$$

If $x \in m+[-\delta / 8, \delta / 8]^{n}$ and $y \in \operatorname{supp} a(|m|(\cdot-m))$, then

$$
x-y \in\left(m+[-\delta / 8, \delta / 8]^{n}\right)-\left(m+[-\delta / 8, \delta / 8]^{n}\right)=[-\delta / 4, \delta / 4]^{n},
$$

and so $\Psi(x-y)=1$. Hence,

$$
\begin{aligned}
\left.\left.\sum_{m \neq 0} \int_{m+[-\delta / 8, \delta / 8]^{n}}\left|\int_{\mathbb{R}^{n}} e^{-2 \pi i k \cdot y} \Psi(x-y) c_{m}\right| m\right|^{n / p} a(|m|(y-m)) d y\right|^{p} d x \\
=\left.\left.\sum_{m \neq 0} \int_{m+[-\delta / 8, \delta / 8]^{n}}\left|\int_{\mathbb{R}^{n}} e^{-2 \pi i k \cdot y} c_{m}\right| m\right|^{n / p} a(|m|(y-m)) d y\right|^{p} d x \\
=\left.\left.\sum_{m \neq 0} \int_{m+[-\delta / 8, \delta / 8]^{n}}\left|c_{m}\right| m\right|^{n / p}|m|^{-n} \widehat{a}(k /|m|)\right|^{p} d x \\
=(\delta / 4)^{n} \sum_{m \neq 0}\left|c_{m}\right|^{p}|m|^{n-p n}|\widehat{a}(k /|m|)|^{p} .
\end{aligned}
$$

Moreover, using $|\widehat{a}(\xi)| \geq c>0$ for all $1 / 2 \leq|\xi| \leq 2$, we obtain

$$
\begin{aligned}
\left\|\left(M_{k} \Psi\right) * f\right\|_{L^{p}}^{p} \geq(\delta / 4)^{n} \sum_{m \neq 0}\left|c_{m}\right|^{p}|m|^{n-p n}|\widehat{a}(k /|m|)|^{p} \\
\geq(\delta / 4)^{n} \sum_{|k| / 2 \leq|m| \leq 2|k|}\left|c_{m}\right|^{p}|m|^{n-p n}|\widehat{a}(k /|m|)|^{p} \\
\geq C \sum_{|k| / 2 \leq|m| \leq 2|k|}\left|c_{m}\right|^{p}|m|^{n-p n} \geq C|k|^{n-p n} \sum_{|k| / 2 \leq|m| \leq 2|k|}\left|c_{m}\right|^{p}
\end{aligned}
$$

for all $k \neq 0$. Then

$$
\begin{aligned}
\|f\|_{M_{s}^{p, q}} & \asymp\left(\sum_{k \in \mathbb{Z}^{n}}(1+|k|)^{s q}\left\|\left(M_{k} \Psi\right) * f\right\|_{L^{p}}^{q}\right)^{1 / q} \\
& \geq C\left\{\sum_{k \neq 0}(1+|k|)^{s q}\left(|k|^{n-p n} \sum_{|k| / 2 \leq|m| \leq 2|k|}\left|c_{m}\right|^{p}\right)^{q / p}\right\}^{1 / q} \\
& \geq C\left\{\sum_{k \neq 0}|k|^{(n(1 / p-1)+s) q}\left(\sum_{|k| / 2 \leq|m| \leq 2|k|}\left|c_{m}\right|^{p}\right)^{q / p}\right\}^{1 / q} .
\end{aligned}
$$


Therefore, by our assumption $h^{p} \hookrightarrow M_{s}^{p, q},(4.12)$ and (4.14),

$$
\begin{aligned}
\left\{\sum_{k \neq 0}|k|^{(n(1 / p-1)+s) q}\right. & \left.\left.\sum_{|k| / 2 \leq|m| \leq 2|k|}\left|c_{m}\right|^{p}\right)^{q / p}\right\}^{1 / q} \\
& \leq C_{1}\|f\|_{M_{s}^{p, q}} \leq C_{2}\|f\|_{h^{p}} \leq C_{3}\left(\sum_{l \neq 0}\left|c_{l}\right|^{p}\right)^{1 / p}
\end{aligned}
$$

REMARK 4.5. For $f$ defined by (4.11) with $p=1$ to belong to $L^{1}\left(\mathbb{R}^{n}\right)$ (instead of $h^{1}\left(\mathbb{R}^{n}\right)$ ), we do not need the condition $\int_{\mathbb{R}^{n}} x^{\alpha} a(x) d x=0$ for $|\alpha| \leq[n(1 / p-1)]$. Let $f$ be defined by (4.11) with $p=1$, and let $a \in \mathcal{S}\left(\mathbb{R}^{n}\right)$ with $\operatorname{supp} a \subset[-\varepsilon, \varepsilon]^{n},\|a\|_{L^{\infty}} \leq 1$ and $|\widehat{a}(\xi)| \geq c>0$ for all $|\xi| \leq 2$ (see the proof of Lemma 4.3 for the existence of such a function). As in the proof of Lemma 4.4 , we can prove that, if $L^{1}\left(\mathbb{R}^{n}\right) \hookrightarrow M_{s}^{1, q}\left(\mathbb{R}^{n}\right)$, then there exists a constant $C>0$ such that

$$
\left\{\sum_{k \neq 0}|k|^{s q}\left(\sum_{|l| \geq|k| / 2}\left|c_{l}\right|\right)^{q}\right\}^{1 / q} \leq C \sum_{k \neq 0}\left|c_{k}\right|
$$

for all finitely supported sequences $\left\{c_{k}\right\}_{k \in \mathbb{Z}^{n} \backslash\{0\}}$. In fact, it is enough to modify (4.12) and (4.13).

Proof of Theorem 1.2. We first prove that $h^{p} \hookrightarrow M_{-n(1 / p+1 / q-1)}^{p, q}$ if $p \leq q$. Note that, if $0<p \leq 1$ and $p \leq q$, then $\|f+g\|_{M_{s}^{p, q}}^{p} \leq\|f\|_{M_{s}^{p, q}}^{p}+\|g\|_{M_{s}^{p, q}}^{p}$ for all $f, g \in M_{s}^{p, q}$. Let $f=\sum_{i=1}^{\infty} \lambda_{i} a_{i} \in h^{p}$, where $\left\{a_{i}\right\}$ is a collection of $h^{p}$-atoms and $\left\{\lambda_{i}\right\}$ is a sequence of complex numbers with $\sum_{i=1}^{\infty}\left|\lambda_{i}\right|^{p}<\infty$. By Lemmas 4.1 and 4.2 , if $p \leq q$, and if $q \leq 2$ or $q=\infty$, then

$$
\|f\|_{M_{-n(1 / p+1 / q-1)}^{p, q}}^{p} \leq \sum_{i=1}^{\infty}\left|\lambda_{i}\right|^{p}\left\|a_{i}\right\|_{M_{-n(1 / p+1 / q-1)}^{p, q}}^{p} \leq C \sum_{i=1}^{\infty}\left|\lambda_{i}\right|^{p},
$$

and (2.5) gives $\|f\|_{M_{-n(1 / p+1 / q-1)}^{p, q}} \leq C\|f\|_{h^{p}}$, that is, $h^{p} \hookrightarrow M_{-n(1 / p+1 / q-1)}^{p, q}$ if $p \leq q$, and if $q \leq 2$ or $q=\infty$. By interpolation, the embeddings $h^{p} \hookrightarrow$ $M_{-n(1 / p-1 / 2)}^{p, 2}$ and $h^{p} \hookrightarrow M_{-n(1 / p-1)}^{p, \infty}$ imply $h^{p} \hookrightarrow M_{-n(1 / p+1 / q-1)}^{p, q}$ with $2<$ $q<\infty$. Hence, $h^{p} \hookrightarrow M_{-n(1 / p+1 / q-1)}^{p, q}$ if $p \leq q$.

In the case $p \leq q$, since $h^{p} \hookrightarrow M_{-n(1 / p+1 / q-1)}^{p, q}$ and $M_{-n(1 / p+1 / q-1)}^{p, q} \hookrightarrow$ $M_{s}^{p, q}$ if $s \leq-n(1 / p+1 / q-1)$, we see that condition (d) gives $h^{p} \hookrightarrow M_{s}^{p, q}$. In the case $p>q$, since $h^{p} \hookrightarrow M_{-n(2 / p-1)}^{p, p}$ and $M_{-n(2 / p-1)}^{p, p} \hookrightarrow M_{s}^{p, q}$ if $s<$ $-n(1 / p+1 / q-1)$ (Lemma 2.2 ), we also see that condition (c) gives $h^{p} \hookrightarrow$ $M_{s}^{p, q}$.

We next prove that the embedding $h^{p} \hookrightarrow M_{s}^{p, q}$ implies (c) or (d). Assume that $h^{p} \hookrightarrow M_{s}^{p, q}$. Then, from Lemma 4.4, 


$$
\left\{\sum_{k \neq 0}|k|^{(n(1 / p-1)+s) q}\left(\sum_{|k| / 2 \leq|l| \leq 2|k|}\left|c_{l}\right|^{p}\right)^{q / p}\right\}^{1 / q} \leq C\left(\sum_{k \neq 0}\left|c_{k}\right|^{p}\right)^{1 / p}
$$

for all $\left\{c_{k}\right\}_{k \in \mathbb{Z}^{n} \backslash\{0\}} \in \ell^{p}$, where we have used the limit argument. If we choose the sequence $\left\{c_{k}\right\}_{k \in \mathbb{Z}^{n} \backslash\{0\}}$ defined by $c_{k}=1$ when $|k| \leq N$ and $c_{k}=0$ when $|k|>N$, where $N$ is a positive integer satisfying $N \geq 2$, then (4.16) implies

$$
\begin{aligned}
C N^{n / p} & \geq C\left(\sum_{0<|k| \leq N} 1\right)^{1 / p}=C\left(\sum_{k \neq 0}\left|c_{k}\right|^{p}\right)^{1 / p} \\
& \geq\left\{\sum_{k \neq 0}|k|^{(n(1 / p-1)+s) q}\left(\sum_{|k| / 2 \leq|l| \leq 2|k|}\left|c_{l}\right|^{p}\right)^{q / p}\right\}^{1 / q} \\
& \geq\left\{\sum_{0<|k| \leq N / 2}|k|^{(n(1 / p-1)+s) q}\left(\sum_{|k| / 2 \leq|l| \leq 2|k|}\left|c_{l}\right|^{p}\right)^{q / p}\right\}^{1 / q} \\
& =\left\{\sum_{0<|k| \leq N / 2}|k|^{(n(1 / p-1)+s) q}\left(\sum_{|k| / 2 \leq|l| \leq 2|k|} 1\right)^{q / p}\right\}^{1 / q} \\
& \geq C\left\{\sum_{0<|k| \leq N / 2}|k|^{(n(2 / p-1)+s) q}\right\}^{1 / q} \\
& \geq C N^{n(2 / p-1)+s+n / q} .
\end{aligned}
$$

Therefore, $p, q, n$ must satisfy $n(2 / p-1)+s+n / q \leq n / p$, that is, $s \leq$ $-n(1 / p+1 / q-1)$.

In the case $p \leq q$, we have nothing to prove any more, that is, we have (d). Let $p>q$, and assume that $s \geq-n(1 / p+1 / q-1)$. We can take $\varepsilon>0$ such that $(1+\varepsilon) q / p<1$. We define $\left\{c_{k}\right\}_{k \in \mathbb{Z}^{n} \backslash\{0\}}$ by

$$
c_{k}= \begin{cases}|k|^{-n / p}(\log |k|)^{-(1+\varepsilon) / p} & \text { if }|k| \geq N, \\ 0 & \text { if }|k|<N,\end{cases}
$$

where $N$ is a sufficiently large integer. Note that $\left\{|k|^{-n / r}(\log |k|)^{-\alpha / r}\right\}_{|k| \geq N}$ $\in \ell^{r}$ if $\alpha>1$, and $\left\{|k|^{-n / r}(\log |k|)^{-\alpha / r}\right\}_{|k| \geq N} \notin \ell^{r}$ if $\alpha \leq 1$ (see, for example, $[15$, Remark 4.3]). Thus,

$$
\left(\sum_{k \neq 0}\left|c_{k}\right|^{p}\right)^{1 / p}=\left\{\sum_{|k| \geq N}\left(|k|^{-n / p}(\log |k|)^{-(1+\varepsilon) / p}\right)^{p}\right\}^{1 / p}<\infty .
$$

On the other hand,

$$
\left\{\sum_{k \neq 0}|k|^{(n(1 / p-1)+s) q}\left(\sum_{|k| / 2 \leq|l| \leq 2|k|}\left|c_{l}\right|^{p}\right)^{q / p}\right\}^{1 / q}=\infty .
$$


In fact, since $n(1 / p-1)+s \geq-n / q$ and $(1+\varepsilon) q / p<1$, we see that

$$
\begin{aligned}
\left\{\sum_{k \neq 0} \mid\right. & \left.\left.k\right|^{(n(1 / p-1)+s) q}\left(\sum_{|k| / 2 \leq|l| \leq 2|k|}\left|c_{l}\right|^{p}\right)^{q / p}\right\}^{1 / q} \\
& \geq\left\{\sum_{|k| \geq 2 N}|k|^{(n(1 / p-1)+s) q}\left(\sum_{|k| / 2 \leq|l| \leq 2|k|}\left(|l|^{-n / p}(\log |l|)^{-(1+\varepsilon) / p}\right)^{p}\right)^{q / p}\right\}^{1 / q} \\
& \geq C\left\{\sum_{|k| \geq 2 N}|k|^{(n(1 / p-1)+s) q}(\log |k|)^{-(1+\varepsilon) q / p}\right\}^{1 / q} \\
& \geq C\left\{\sum_{|k| \geq 2 N}\left(|k|^{-n / q}(\log |k|)^{-\{(1+\varepsilon) q / p\} / q}\right)^{q}\right\}^{1 / q}=\infty
\end{aligned}
$$

However, (4.17) and (4.18) contradict (4.16). Consequently, $p, q, s$ must satisfy $s<-n(1 / p+1 / q-1)$, that is, we have (c).

We end this paper by giving the following remark which we already mentioned in the Introduction.

REMARK 4.6. Let $1 \leq q<\infty$. As in the proof of Theorem 1.2 , we can prove that, if $L^{1}\left(\mathbb{R}^{n}\right) \hookrightarrow M_{s}^{1, q}\left(\mathbb{R}^{n}\right)$, then $s<-n / q$. In fact, if $L^{1} \hookrightarrow M_{s}^{1, q}$, then we have (4.15) (Remark 4.5). By choosing $c_{k}=|k|^{-n}(\log |k|)^{-(1+\varepsilon)}$ for $|k|$ large, where $\varepsilon>0$ satisfies $\varepsilon q<1$, we see that (4.15) holds only if $s<-n / q$.

\section{References}

[1] J. Duoandikoetxea, Fourier Analysis, Grad. Stud. Math. 29, Amer. Math. Soc., Providence, RI, 2001.

[2] H. G. Feichtinger, Banach spaces of distributions of Wiener's type and interpolation, in: P. L. Butzer et al. (eds.), Functional Analysis and Approximation (Oberwolfach, 1980), Int. Ser. Numer. Math. 60, Birkhäuser, Basel, 1981, 153-165.

[3] - Modulation spaces on locally compact abelian groups, in: M. Krishna et al. (eds.), Wavelets and their Applications (Chennai), Allied Publ., New Delhi, 2003, 99-140 (updated version of a technical report, Univ. of Vienna, 1983).

[4] - Modulation spaces: looking back and ahead, Sampl. Theory Signal Image Process. 5 (2006), 109-140.

[5] M. Frazier and B. Jawerth, Decomposition of Besov spaces, Indiana Univ. Math. J. 34 (1985), 777-799.

[6] D. Goldberg, A local version of real Hardy spaces, Duke Math. J. 46 (1979), 27-42.

[7] P. Gröbner, Banachräume glatter Funktionen und Zerlegungsmethoden, thesis, Univ. of Vienna, 1992.

[8] K. Gröchenig, Foundations of Time-Frequency Analysis, Birkhäuser, Boston, 2001.

[9] —, Time-frequency analysis of Sjöstrand's class, Rev. Mat. Iberoamer. 22 (2006), 703-724. 
[10] M. Kobayashi, Modulation spaces $M^{p, q}$ for $0<p, q \leq \infty$, J. Funct. Spaces Appl. 4 (2006), 329-341.

[11] M. Kobayashi and Y. Sawano, Molecular decomposition of the modulation spaces $M_{p, q}^{s}$, preprint.

[12] K. Okoudjou, Embedding of some classical Banach spaces into modulation spaces, Proc. Amer. Math. Soc. 132 (2004), 1639-1647.

[13] W. Sickel and H. Triebel, Hölder inequalities and sharp embeddings in function spaces of $B_{p q}^{s}$ and $F_{p q}^{s}$ type, Z. Anal. Anwend. 14 (1995), 105-140.

[14] M. Sugimoto and N. Tomita, The dilation property of modulation spaces and their inclusion relation with Besov spaces, J. Funct. Anal. 248 (2007), 79-106.

[15] M. Sugimoto and N. Tomita, A remark on fractional integrals on modulation spaces, Math. Nachr. 281 (2008), 1372-1379.

[16] J. Toft, Continuity properties for modulation spaces, with applications to pseudodifferential calculus, I, J. Funct. Anal. 207 (2004), 399-429.

[17] H. Triebel, Modulation spaces on the Euclidean n-space, Z. Anal. Anwend. 2 (1983), 443-457.

[18] —, Theory of Function Spaces, Birkhäuser, Basel, 1983.

[19] B. Wang and C. Huang, Frequency-uniform decomposition method for the generalized BO, KdV and NLS equations, J. Differential Equations 239 (2007), 213-250.

[20] B. Wang and H. Hudzik, The global Cauchy problem for the NLS and NLKG with small rough data, ibid. 232 (2007), 36-73.

Masaharu Kobayashi

Department of Mathematics

Tokyo University of Science

Kagurazaka 1-3, Shinjuku-ku

Tokyo 162-8601, Japan

E-mail: kobayashi@jan.rikadai.jp

Naohito Tomita

Department of Mathematics

Graduate School of Science

Osaka University

Toyonaka, Osaka 560-0043, Japan

E-mail: tomita@math.sci.osaka-u.ac.jp
Akihiko Miyachi Department of Mathematics Tokyo Woman's Christian University Zempukuji, Suginami-ku Tokyo 167-8585, Japan E-mail: miyachi@lab.twcu.ac.jp

Received August 19, 2008

Revised version October 20, 2008 